\title{
Phosphoketolase activity in ruminal butyrivibrios
}

\author{
M Marounek, D Duskova \\ Institute of Animal Physiology and Genetics, Czech Academy of Sciences, \\ Prague 10, Uhrineves, Czech Republic
}

\begin{abstract}
Few data are available on the intracellular metabolism of pentoses in rumen bacteria. Some non-rumen bacteria and yeasts convert pentoses to acetyl phosphate via xylulose-5phosphate through the action of phosphoketolase (EC 4.1.2.9). Radioisotope studies indicate that this metabolic pathway has physiological significance in the rumen (Wallnöfer et al, 1966, Appl Microbiol, 14, 1004-1010). Rumen microbial species with this activity remain to be identified. Phosphoketolase was absent in Prevotella ruminicola B14 and Fibrobacter succinogenes S85 (Matte et al, 1992, Can J Microbiol, 38, 370-376).
\end{abstract}

In our study, the activity of phosphoketolase and lactate dehydrogenase (LDH) were determined in Butyrivibrio fibrisolvens ATCC 19171 (the type strain) and in $B$. fibrisolvens strains $86, \mathrm{X} 1, \mathrm{X} 2 \mathrm{D} 62$ and CE51. Phosphoketolase was determined according to Matte et al (1992) and LDH using a commercial kit. Lactobacillus plantarum 185 was used as a positive control. All bacterial strains showed a significant LDH activity, both in glucose- and xylose-grown cells. B. fibrisolvens CE51 possessed the phosphoketolase activity in xylose-grown cells : $12.1 \mathrm{nmol} . \mathrm{min}^{-1} \cdot \mathrm{mg}^{-1}$ protein. In this strain, the composition of fermentation end-products differed significantly in cultures supplied with glucose and xylose. lodoacetate $(3 \mathrm{mM})$ had no effect on liberation of acetyl phosphate from xyluloso-5phosphate. In other butyrivibrios the phosphoketolase activity was absent and metabolic profiles were the same on both carbon sources. Activity of phosphoketolase in L. plantarum 185 was 33.7 and $21.9 \mathrm{nmol}$. $\mathrm{min}^{-1} . \mathrm{mg}^{-1}$ on glucose and xylose, resp. Phosphoketolase was absert in pectinolytic $B$. fibrisolvens 787 grown on glucose and pectin.

In mixed cultures of rumen microorganisms $75 \%$ of the xylose was converted to hexose via the route involving transketolase and transaldolase and $25 \%$ was metabolized through the action of phosphoketolase (Wallnöfer et al, 1966). It is thus possible that more rumen microorganisms with this activity exist. 